\title{
Multi Step Transient Hybrid Simulation Framework Design and Practical Engineering Application for AC I DC Power Grid
}

\author{
Zheng Weijie ${ }^{1}$, Xu Dechao ${ }^{1 *}$, Chen Yong $^{1}$, Zhang Xing $^{1}$, Mu Qing ${ }^{1}$ \\ ${ }^{1}$ CEPRI, Beijing, Province, 100192, China
}

\begin{abstract}
Based on the large power grid simulation and the existing security prevention and control mode, this paper further integrates the multi-step transient hybrid simulation architecture computing technology to research the high-performance analysis and situational awareness simulation technology of interconnected AC / DC large power grid security and stability characteristics. So as to realize the "panoramic situational awareness, wide area coordinated control, flexible and efficient service" of large power grid, and improve the super large scale power grid Comprehensive security defense and intelligent monitoring level of panoramic, multi-dimensional and three-dimensional.
\end{abstract}

\section{Introduction}

As the increasing proportion of UHVDC transmission, the AC-DC coupling characteristics of power grid are more significant. In order to adapt to the characteristics of the current power grid, it is necessary to increase the scale of electromagnetic network in electromechanical electromagnetic hybrid simulation. Therefore, new and higher requirements are put forward for electromechanical electromagnetic hybrid simulation technology[1-3]. It is necessary to research the parallel algorithm of electromagnetic electromechanical hybrid simulation based on electromechanical electromagnetic interface boundary point decoupling, to realize the hybrid simulation of electromagnetic multi subnet parallel and electromechanical multi subnet parallel, and greatly improve the parallel degree of hybrid simulation.

At present, the State Grid Corporation of China (SGCC) is actively implementing the "west to East power transmission", "North South mutual supply" and "National Networking" projects to jointly form an UHV power grid covering large power supply bases and load centers, and promote cross provincial and cross regional power transactions[4-6]. So as to realize the optimal allocation of energy resources in a wide range and improve the overall economic benefits of the power grid. With the increasing scale of interconnected power grid, considering the security and stability of local power grid in isolation can not meet the requirements of actual large-scale interconnected system analysis, and reliable large-scale power grid simulation tools are needed. At the same time, the wide application of high-power power electronic equipment (such as DC transmission, SVC, TCSC) and various fast automatic control devices also make the dynamic characteristics of power grid more stable with the increasing complexity, traditional electromechanical transient simulation has been difficult to accurately simulate the dynamic characteristics of power grid.

\section{Multi Step Transient Hybrid Simulation Framework Design}

Security and stability is the primary task of power grid operation. The modern society is increasingly dependent on power, which requires higher requirements for safe and stable operation of large power grid and intelligent prevention and control. It is necessary to develop and establish online security analysis and control support system [2-3]. At present, the electromechanical transient simulation method is used in all the online analysis systems in the world. The online application of electromechanical electromagnetic hybrid simulation has not been reported, especially in the current large-scale electromagnetic environment, the speed, accuracy and automation of hybrid simulation can not meet the online application conditions[7-9]. The purpose of this paper is to study the online operation requirements of Large-scale Electromechanical electromagnetic hybrid simulation, and realize online super real-time electromechanical electromagnetic hybrid simulation supporting electromagnetic model of multi DC system. 


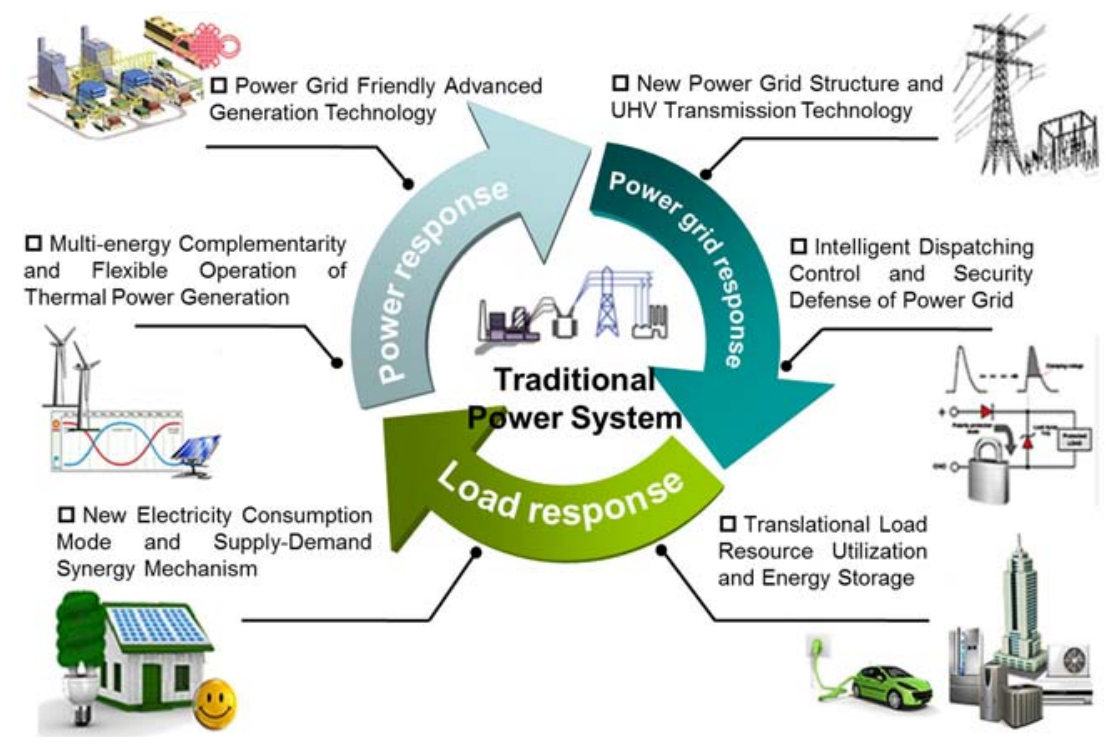

Figure 1. Display effect picture of panoramic simulation system

The main key technologies of the design architecture are as follows:

1) the research on the super large scale parallel simulation of electromechanical electromagnetic, and the fine-grained parallel method is adopted to improve the maximum parallelism bottleneck of hybrid simulation, and greatly reduce the computation time.
2) The research and development of electromagnetic characteristics simulation algorithm on the mechanical and electrical side can solve the problem that the current electromechanical simulation can not deal with non power frequency signals, and realize the simulation calculation of electromechanical electromagnetic fusion with high precision and high speed.

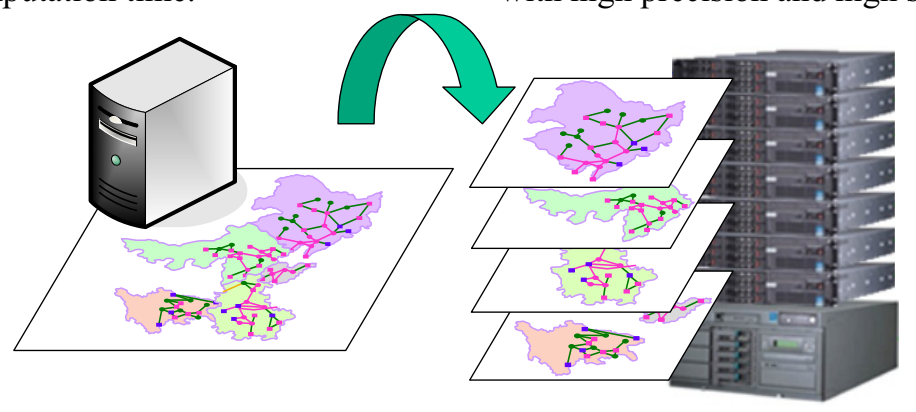

Figure 2. Physical architecture diagram of high performance parallel computing

3) The intelligent pretreatment technology of electromagnetic model can reduce the preparation time of electromagnetic simulation calculation and support the demand of online application. The hybrid simulation of electromechanical electromagnetic is realized online. Finally, the on-line super real-time electromagnetic electromechanical simulation of complex large power grid will be realized. After the technical function is put into operation, it will effectively improve the accuracy of the simulation of AC / DC power grid in China and realize the on-line practicability of the electromechanical electromagnetic hybrid simulation function.

ADPSS uses node splitting method to split boundary nodes to solve hybrid simulation interface circuit[7-11]. As shown in the figure below, $\mathrm{A}$ is electromagnetic network and $\mathrm{B}$ is electromechanical network. YA and $\mathrm{YB}$ are the node conductance matrices of $\mathrm{A}$ and $\mathrm{B}$ respectively, and $\mathrm{hA}$ and $\mathrm{hB}$ are the equivalent current sources of $\mathrm{A}$ and $\mathrm{B}$ respectively. The broken line between the tearing boundary nodes flows current i $\alpha$, and the dimension is the number of boundary points $\mathrm{N}$. After derivation, the formula for solving current i $\alpha$ is as follows:

$$
\left(p^{T} Y_{A}^{-1} p+Y_{B}^{-1}\right) i_{\alpha}=Y_{B}^{-1} h_{B}-p^{T} Y_{A}^{-1} h_{A}
$$

Where $\mathrm{p}$ and $\mathrm{q}$ are the incidence matrix, which respectively represent the correlation between $\mathrm{i} a$ and the boundary node of electromagnetic network A, and the correlation between $i \mathrm{a}$ and the boundary node of electromechanical network B. once the current $i$ a is obtained, all the injected currents of electromagnetic network $\mathrm{A}$ are known, so the electromagnetic network can be solved. Calculating the current vector $i$ a is the key to solve the electromagnetic network, which takes up most of the hybrid simulation interface calculation[8-13]. 


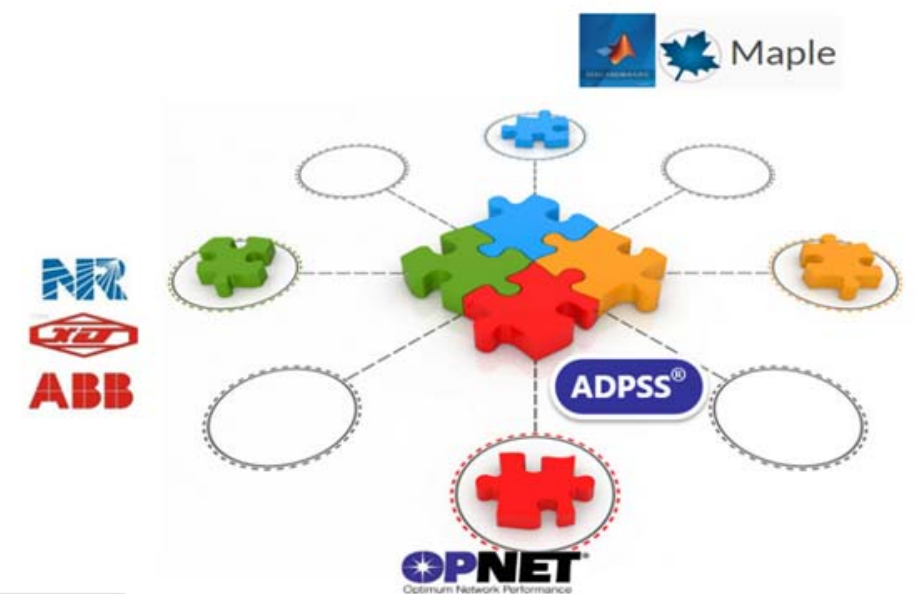

Figure 3. ADPSS open parallel architecture platform

The computational complexity of the electromagnetic side at the hybrid simulation interface can be approximately estimated as follows:

$$
\begin{aligned}
f_{E M T}(N, m) \approx & O\left(N^{3}\right)+(3 N+2) \cdot O(\text { flops }) \\
& +O(m+\text { flops })
\end{aligned}
$$

In the second term of the above formula, the former generation back generation calculation or the matrix multiplication is related to the dimension of the matrix, or is equal to the number of boundary points $3 \mathrm{~N}$ dimension, or is the number of single-phase nodes $\mathrm{m}$ of the associated electromagnetic subnet. The amount of computation on the electromagnetic side of the hybrid simulation interface is proportional to $\mathrm{N} 3[10-14]$.
The calculation of hybrid simulation interface of electromagnetic side is roughly calculated by taking YA as full array in the worst case. According to the calculation complexity of LU decomposition and previous generation of full array, it can be deduced as follows:

$$
f_{E M T}(N, m) \approx N^{3}+(3 N+2) \cdot\left(m^{2}+m\right)+\frac{m^{3}}{3}
$$

The electromagnetic side sub network group includes several electromagnetic transient calculation subnets, which are connected with the sub network through crosssection. See the parallel computing architecture of the subnet group.

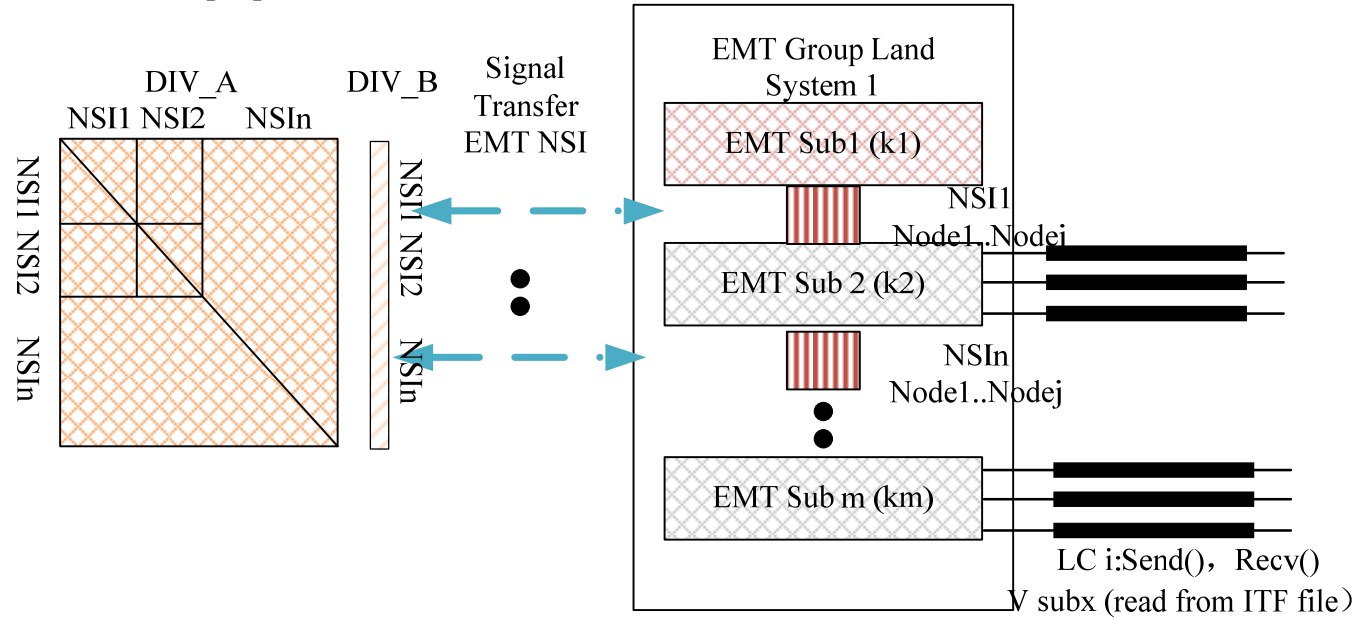

EMT_Group

Main Control

EMT_Group

Normal Sub

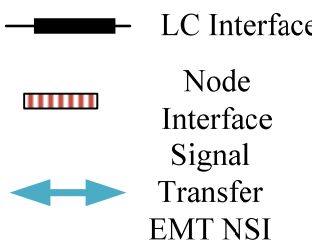

Figure 4. Comparison Parallel simulation framework within EMT multi time scale subnet cluster 
Section (DIV) is the direct connection surface between one subnet and another subnet through a certain interface, which is expressed as DIV 1 to DIVN. Each individual subnet may contain four different types of sections. The four types of interfaces are st interface, SLC interface, LC interface and node interface. Each section has the unique section number of the whole network and the unique section number of the whole subnet group. At the same time, each section also includes the unique section number of the whole network and the unique section number of the whole subnet group.

\section{Verification and confirmation}

Each section contains a certain number of interface elements (ITF). Nsi1 interface contains Node1.. Nodej, $\mathrm{j}$ nodes as interfaces; LCi interface contains an LC element. Through the file mapping table in the subnet, the interface components are mapped to the components in the subnet, such as LC transmission line, single-phase transmission line and electrical Node.

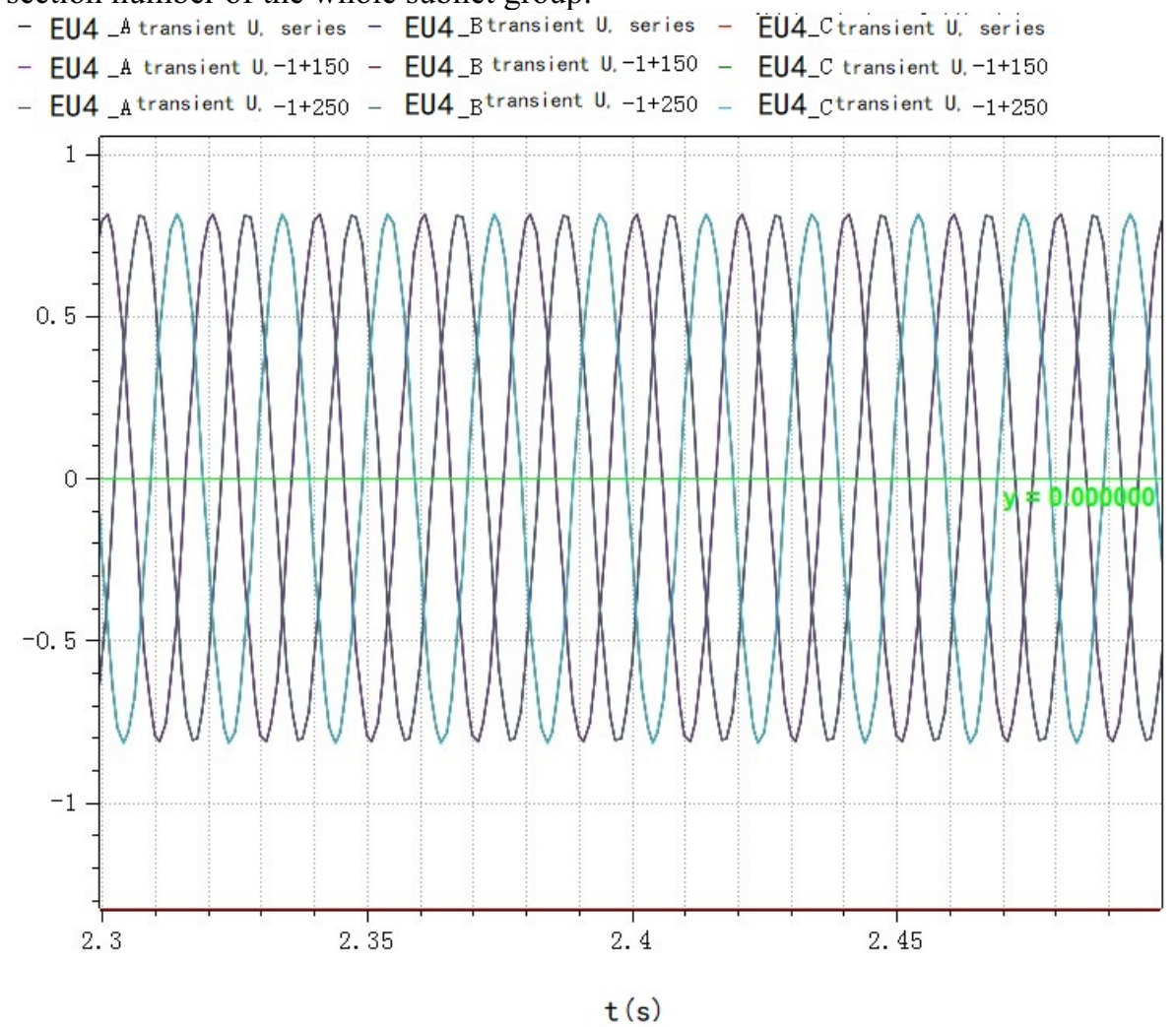

Figure 5. Comparison of electromagnetic calculation results of large power grid hybrid simulation cases

In order to test the performance of the algorithm under different heterogeneity levels of nodes, the experiment set 500 nodes in the cluster, in which the CPU and memory available resources obey the mean value of 50 and 10 respectively, and the coefficient of variation increases from 0.00 (fully homogeneous) to 1.0 (heterogeneous) truncated normal distribution with the increment of 0.1 The CPU core resource is integer and the memory resource is floating-point. There are 11 kinds of experimental environments. The heterogeneity of resources increases with the increase of coefficient of variation.

At the same time, the experiment constructed 100 online tasks, in which the number of each task process was set to be 2 to 200 evenly distributed, in which $3 \%$ of the processes were the main control process, and the rest were the computing process. The CPU resource demand of the main control process resource was set to 1 , and the memory demand was set to obey the truncated normal distribution with the average value of 0.3 and the coefficient of variation of 0.1 , and the lower limit value was greater than 0 In resource demand, CPU resource demand is set to 1 , memory demand is set to obey the truncated normal distribution with mean value of 0.05 and coefficient of variation of 0.15 , and the lower limit is greater than 0 .

\section{Acknowledgments}

The paper is supported by Science and Technology Project of SGCC "Research and development of high performance computing technology for power system application" (XTB17201900305)

\section{References}

1. RITTIGER J. Digital simulation of HVDC transmission and its correlation to simulator studies[C]//International Conference on AC and DC Power Transmission, 1991: 414-416. 
2. REEVE J, ADAPA R. A new approach to dynamic analysis of AC networks incorporating detailed modeling of DC systems. I. Principles and implementation[J]. IEEE Transactions on Power Delivery, 1988, 3(4): 2005-2011.

3. ADAPA R, REEVE J. A new approach to dynamic analysis of AC networks incorporating detailed modeling of DC systems. II. Application to interaction of $\mathrm{DC}$ and weak $\mathrm{AC}$ systems[J]. IEEE Transactions on Power Delivery, 1988, 3(4): 20122019.

4. RITTIGER J, LEHN P, KULICKE B. Comparison of the ATP version of the EMTP and the NETOMAC program for simulation of HVDC systems[J]. IEEE Transactions on Power Delivery, 1995, 10(4): 2048-2053.

5. ANDERSON G J, WATSON N R, ARNOLD C P, et al. A new hybrid algorithm for analysis of HVDC and FACTS systems[C]//International Conference on Energy Management and Power Delivery (EMPD), 1995: 462-467.

6. CROW M L, CHEN J-g. The multirate method for simulation of power system dynamics[J]. IEEE Transactions on Power Systems, 1994, 9(3): 16841690.

7. HEFFERNAN M D, TURNER K S, ARRILLAGA J, et al. Computation of AC - DC system DisturbancesPart i. interactive coordination of generator and convertor transient models[J]. IEEE Power Engineering Review, 1981, 1(11): 15-16.

8. MARTI J R, LINARES L R, CALVINO J, et al. OVNI: an object approach to real-time power system simulators[C]//International Conference on Power System Technology (POWERCON), 1998: 977-981.

9. MEER A D, GIBESCU M, MEIJDEN M D, et al. Advanced hybrid transient stability and EMT simulation for VSC-HVDC systems[J]. IEEE Transactions on Power Delivery, 2015, 30(3): 10571066.

10. HUANG Q, VITTAL V. Application of electromagnetic Transient-Transient stability hybrid simulation to fidvr study[J]. IEEE Transactions on Power Systems, 2015, PP(99): 1-13.

11. CROW M L, CHEN J-g. The multirate method for simulation of power system dynamics[J]. IEEE Transactions on Power Systems, 1994, 9(3): 16841690.

12. MARTÍ J, LINARES L, HOLLMAN J A, et al. Ovni:Integrated Software/Hardware solution for Real-Time simulation of large power systems[C]//14th Power Systems Computer Conference (PSCC), 2002: 1-7.

13. ADAPA R, REEVE J. A new approach to dynamic analysis of AC networks incorporating detailed modeling of DC systems. II. Application to interaction of DC and weak AC systems[J]. IEEE Transactions on Power Delivery, 1988, 3(4): 20122019.
14. YUE Cheng-yan, TIAN Fang, ZHOU Xiao-xin, WU Zhong-xi, LI Ruo-mei, Implemention of Interfaces for Hybrid Simulation of Power System Electromagnetic-Electromechanical Transient Process[J]. Power System Technology, 2006, 30(4):6-9. 